\title{
Estimando Indigencia y Pobreza Indígena Regional con Datos Censales y Encuestas de HOGARES*
}

\author{
Claudio A. Agostini \\ Ilades-Universidad Alberto Hurtado \\ PhILIP H. BROWN \\ Colby College \\ ANDREI ROMAN \\ Colby College
}

\begin{abstract}
While indigenous Chileans are believed to experience higher poverty rates than non-indigenous Chileans, evidence is typically based on surveys that are not representative by ethnicity. We adapt poverty mapping methodologies to estimate poverty and poverty gaps for eight indigenous groups more precisely. We find that indigenous people experience higher rates of poverty and also greater depth of poverty. These results hold within regions, suggesting that geographic variation in economic opportunity cannot fully explain the differences. We also find that the poverty burden is not equal across indigenous groups and argue that ethnicity should be included as a criterion for antipoverty targeting.
\end{abstract}

JEL: I32, J15, O18

Keywords: Pobreza, Indigencia, Pueblos Indígenas, Mapas de Pobreza, Chile

\section{INTRODUCCIÓN}

El crecimiento económico experimentado por Chile desde mediados de los años ochenta puede caracterizarse como rápido y sostenido. Es así como entre 1986 y 2005 , por ejemplo, el PIB creció a una tasa promedio de $6 \%$. El crecimiento experimentado en las últimas dos décadas ha sido acompañado por una fuerte reducción en la pobreza: mientras el PIB per cápita aumentó de US\$1.679

\footnotetext{
* Agradecemos a Gabriel Demombynes, Peter Lanjouw, Marcela Perticará, un árbitro anónimo y los participantes de un seminario en Ilades-Universidad Alberto Hurtado por múltiples sugerencias y comentarios. Agradecemos también el apoyo económico de Colby College.

Email: agostini@uahurtado.cl
} 
en 1987 a US\$ 9.879 en 2007, la fracción de la población viviendo bajo la línea oficial de pobreza disminuyó de 39,4\% a 13,7\%. Las tasas de indigencia también cayeron fuertemente en este período, desde $14,2 \%$ a $3,2 \%$. Si bien el crecimiento económico explica una parte importante de la disminución en la pobreza ('Larrañaga,", 2001; Contreras, $2003_{1}^{\prime}$, un conjunto de transferencias monetarias y en especies del gobierno a los hogares pobres ha demostrado ser fundamental en la reducción de la pobreza en Chile (Beyer, 1997; 'Valdés 1999i).

Con menos hogares pobres, la probabilidad de distribuir recursos en forma ineficiente entregándoselos a quiénes no son pobres (es decir, cometer error tipo I) aumenta, haciendo que el criterio para identificar a los hogares pobres sea extremadamente importante y un desafío creciente para las políticas sociales en Chile.

Actualmente, los criterios de elegibilidad para las transferencias gubernamentales están determinados principalmente por el ingreso y algunas características socioeconómicas y demográficas de los hogares. Sin embargo, 'Agostini y Brown (2010a)', encuentran evidencia de potenciales ganancias en la eficiencia de las transferencias en Chile si se incluyeran variables geográficas en los criterios de asignación.

Si bien la evidencia para otros países sugiere que programas para reducir la pobreza basados sólo en criterios geográficos no son muy efectivos ${ }^{1}$, los resultados muestran la necesidad de utilizar criterios complementarios para medir ingreso con el objetivo de mejorar la focalización de los programas para disminuir la pobreza 'Bigman y Fofack, 2000)' Dada la división socioeconómica actual entre la población indígena y la no indígena en Chile, indicadores estadísticamente confiables de pobreza desagregados por pueblo indígena, idealmente a nivel regional, pueden contribuir significativamente a este objetivo.

La población indígena en Chile tiene una larga historia de marginación, la cual continúa traduciéndose en niveles altos de pobreza. 'IValenzuela $(200 \overline{3})$,', por ejemplo, estima que 32,3\% de la población indígena vivía en situación de pobreza en 2000 , comparado con un $20,1 \%$ de la población no indígena. En promedio, los hogares indígenas en Chile ganan menos de la mitad del ingreso que los no indígenas y $65 \%$ de los hogares indígenas se encuentran en los dos quintiles más bajos de la distribución del ingreso. Esta brecha de ingreso es parcialmente explicada por los perfiles ocupacionales de la fuerza de trabajo indígena: $31 \%$ tiene trabajos como mano de obra no calificada y $25 \%$ trabaja en agricultura ('World Bank, 2002 i).

Sin embargo, todas estas cifras son obtenidas a partir de los datos de la Encuesta Nacional de Caracterización Socioeconómica (CASEN), una encuesta trienal realizada por el Ministerio de Planificación (MIDEPLAN). El problema es que si bien CASEN es representativa a nivel nacional y regional y también a nivel urbano y rural para todo el país (Contreras et $\bar{a}$ (Co $200 \overline{1}$ ), no contiene una muestra representativa para cada uno de los ocho pueblos indígenas reconocidos por la ley Chilena: Mapuche, Aymará, Atacameño, Quechua, Rapanui, Colla, Kawashkar,(o

$1 \mathrm{Al}$ respecto puede verse un estudio para Bangladesh de 슬.

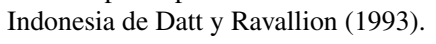


Alacalufe) y Yagán (o Yámana). Incluso más, algunas áreas remotas del país donde viven en proporción significativa algunos pueblos indígenas no están incluidas en la muestra. Como resultado, estimaciones de ingreso y pobreza obtenidos a partir de los datos de la CASEN pueden ser muy imprecisos y no permiten discriminar estadísticamente entre la población indígena y la no indígena (tampoco entre distintos pueblos indígenas o entre poblaciones indígenas en distintas regiones del país).

Para ilustrar este último punto, el Cuadro 1 muestra las tasas de pobreza indígena calculadas por 'Valenzuela $(2003)$ utilizando datos de las encuestas CASEN de 1996 y 2000 y nuestro propio cálculo para el año 2003. La tasa de pobreza para los Kawashkar ha aumentado según estos datos en más de 20 puntos porcentuales hasta un 41,9\%, mientras que la de los Yagán ha disminuido desde $12,3 \%$ a $0 \%$. La pobreza para los dos pueblos indígenas mayoritarios en Chile, Mapuche y Aymará, también ha fluctuado en forma importante con una disminución de cinco puntos porcentuales para los Mapuches y un aumento de nueve puntos porcentuales para los Aymará.

\section{CUADRO 1 \\ TASAS DE POBREZA POR ETNIA CALCULADAS CON ENCUESTA CASEN (Porcentaje)}

\begin{tabular}{lccc}
\hline & 1996 & 2000 & 2003 \\
\hline No-indígena & 22,7 & 20,1 & 22,0 \\
Mapuche & 38,4 & 32,9 & 31,1 \\
Aymará & 23,7 & 32,7 & 27,5 \\
Atacameño & 29,3 & 19,2 & 10,4 \\
Quechua & 27,1 & 25,8 & 17,0 \\
Rapanui & 12,3 & 14,4 & 44,4 \\
Colla & 13,9 & 7,5 & 10,5 \\
Kawashkar & 17,0 & 41,9 & 23,1 \\
Yagán & 12,3 & 0,0 & 0,0 \\
\hline
\end{tabular}

Fuente: 'Valenzuela $(\overline{2} 0 \overline{0} \overline{3})$ y cálculos propios en base a información del Ministerio de Planificación (MIDEPLAN) - CASEN 1996, 2000 y 2003.

Estos cambios dramáticos, la falta de representatividad por etnia de la encuesta y el pequeño tamaño de muestra para algunos pueblos indígenas sugieren que los estimadores de pobreza calculados directamente de la CASEN son poco precisos y probablemente no confiables ${ }^{2}$. Dado que la CASEN es la principal fuente de información sobre ingresos en Chile, esta evidencia presenta un desafío

2 De hecho, el error estándar asociado a cada tasa de pobreza es lo suficientemente alto para que no sea posible rechazar estadísticamente la hipótesis de que la pobreza es la misma en todos los puebles indígenas y que no ha cambiado en el tiempo. 
de política importante: la falta de una clara comprensión de la pobreza indígena y su profundidad entre distintos pueblos indígenas es un obstáculo significativo para mejorar la focalización de programas para reducir la pobreza. Una solución a este problema consistiría en incluir una muestra representativa de cada pueblo indígena en la encuesta CASEN. Sin embargo, el tamaño de muestra necesario y la distribución geográfica de algunos pueblos indígenas hacen que el costo de hacerlo sea demasiado alto.

En este trabajo se utilizan los avances recientes en metodologías de mapas de pobreza para proponer una solución distinta. Específicamente, se combinan datos de ingresos provenientes de la encuesta CASEN con datos detallados respecto a demografía, características de las viviendas y propiedad de activos, provenientes del Censo Nacional de Población y Vivienda, para obtener estimadores precisos y confiables de pobreza e indigencia para cada uno de los pueblos indígenas en Chile, tanto a nivel nacional como regional.

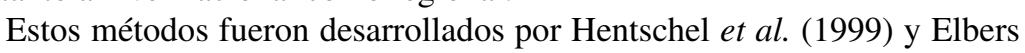
extal. $(2003)$ y han sido utilizados en forma extensa en la literatura reciente para generar mapas espaciales de pobreza en países en desarrollo3.

Los resultados obtenidos en este trabajo muestran que personas pertenecientes a pueblos indígenas son en promedio más pobres que los pertenecientes a la población no indígena y este resultado se mantiene al considerar por separado zonas urbanas, rurales y la capital del país (Santiago). Adicionalmente, las estimaciones muestran que los pueblos Mapuche y Aymará tienen en general las tasas de pobreza más altas. Si bien la ubicación geográfica de cada pueblo indígena puede jugar un rol en explicar las tasas de pobreza más altas, los resultados también muestran que la pobreza indígena es superior a la de la población no indígena en la gran mayoría de las regiones. Basados en esta evidencia, creemos que es posible obtener ganancias significativas en la lucha contra la pobreza si se incluyen variables étnicas como indicadores adicionales para identificar a los hogares elegibles para recibir transferencias gubernamentales. En forma complementaria, dados los resultados de las estimaciones por región, puede ser relevante también incorporar las tasas de pobreza e indigencia indígena regional como una variable más en los criterios de asignación de recursos para transferencias gubernamentales que hace el gobierno central a cada gobierno regional.

Este artículo se organiza de la siguiente forma: la Sección 2 describe la metodología para estimar pobreza por etnia y presenta una revisión de la literatura relevante en la cual se basa; la Sección 3 presenta una breve descripción de las condiciones socioeconómicas de la población indígena en Chile y el contexto de políticas públicas contra la pobreza; la Sección 4 describe los datos de la encuesta CASEN y el censo que son utilizados en este trabajo; la Sección 5 analiza los resultados; y finalmente, la Sección 6 concluye.

\footnotetext{
3 Por ejemplo, 'De- - - - niveles desagregados en Sudáfrica. Elbers et al. (2007)' hacen lo mismo para Mozambique, Madagascar, Ecuador y Camboya.'A y'Ágostini, Brown y Andrei (2010)i los usan para estimar desigualdad a nivel de etnias en Chile.
} 


\section{La Metodología de los Mapas de Pobreza}

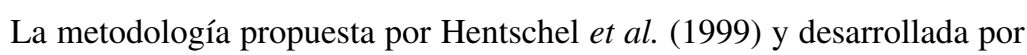
El tiene un censo y la información detallada, incluyendo los ingresos, que dispone una encuesta de hogares. En esta sección entregamos una explicación resumida que permite entender la metodología y en el Apéndice se presentan los detalles de la misma ${ }^{4}$.

La metodología consiste básicamente en un procedimiento en dos etapas para imputar los datos faltantes de ingreso al censo y luego estimar indicadores de pobreza a un nivel más desagregado que el que permitiría la encuesta, en este caso a nivel de etnias.

Para ello, primero se utiliza la encuesta de hogares para estimar la distribución conjunta del ingreso de los hogares y un vector de variables explicativas. Restringiendo el conjunto de variables explicativas a las que estén también disponibles en el censo, los estimadores obtenidos en esta primera etapa se utilizan para estimar la distribución de ingreso para cada pueblo indígena, condicional en las características observables de los individuos y hogares pertenecientes a cada etnia. La forma más simple de estimar el modelo de primera etapa es mediante una aproximación lineal de la expectativa condicional del ingreso de los hogares, permitiendo la presencia de efectos geográficos y heterocedasticidad en la distribución del término de error. El resultado de esta estimación de primera etapa consiste en un vector de coeficientes, una matriz de varianza-covarianza asociada a este vector y un set de parámetros que describen la distribución de los términos de error en la regresión.

La segunda etapa utiliza este set de parámetros junto con las características observables de los individuos y los hogares en el censo con el objeto de generar valores predichos de ingreso y de los errores que no son observados. Para estos efectos, se realiza un bootstrap que simula los valores del ingreso de cada hogar.

Finalmente, el set completo de valores simulados se utiliza para calcular el valor esperado de los indicadores de pobreza para cada pueblo indígena. Este procedimiento es repetido 200 veces, utilizando un nuevo set de coeficientes y errores en cada simulación. Para cada pueblo indígena se calcula la media y la desviación estándar de los indicadores de pobreza sobre el total de simulaciones, que constituyen las estimaciones punto del indicador y los errores estándar de estos estimadores respectivamente.

Es importante mencionar que un supuesto fundamental detrás de esta metodología es que el modelo estimado usando los datos de la encuesta de hogares es también aplicable a los datos del censo. En este caso particular, la encuesta se realizó en Octubre de 2003 y el Censo en abril de 2002, por lo que la cercanía en el tiempo nos hace creer que el supuesto se debiera cumplir en forma razonable en este caso.

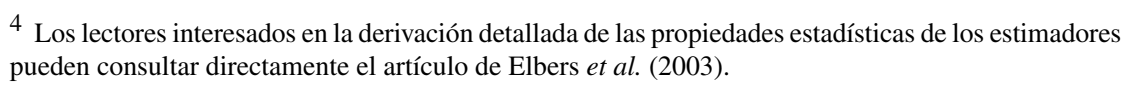




\section{Pueblos Indígenas en Chile}

Los pueblos indígenas representan alrededor de 700,000 hogares $(4,6 \%$ del total) en el Censo 2002. Si bien esto muestra una disminución de alrededor de $30 \%$ en la población indígena respecto al Censo de 1992 cuando era 9,6\% del total, hay bastante consenso en que hubo sobre-representación de los pueblos indígenas en 1992 y la disminución se debe al cambio en la formulación de la pregunta hacia una que captura con más precisión la etnicidad de las personas ('Sanderson, 2005-1) ${ }^{5}$. De hecho, la modificación de la pregunta entre 1992 y 2002 tuvo por objetivo principal evitar que personas que simpatizan con alguna cultura indígena particular o con la causa indígena en general, se declararan como indígenas en el censo ${ }^{6}$.

Si bien es posible que aún existan errores de medición, los datos del Censo de 2002 son considerados más precisos respecto a la cuantificación de la población indígena y son consistentes con otros análisis estadísticos post-censales (STanderson, 2005'). De acuerdo a estos datos, tal como se muestra en el Cuadro 2, hay 3 grupos indígenas mayoritarios que representan cada uno más del $0,1 \%$ de la población total en Chile: Mapuche (que corresponden al 95\% de la población indígena total), Aymará y Atacameño. Sumados, los pueblos Quechua, Rapanui, Colla, Kawashkar, y Yagán abarcan considerablemente menos del 0,2\% del total de la población, y las etnias Kawashkar y Yagán están consideradas en "peligro de extinción" por parte del gobierno chileno que se ha propuesto impedir una mayor disminución en la población de estos pueblos (Gobierno de Chile, $20 \overline{0} \overline{4}$ ). Al igual que la población chilena no indígena, los pueblos indígenas son mayoritariamente urbanos. Entre los Mapuches, quienes tienen la menor tasa de urbanización, menos de $40 \%$ de los hogares se encuentra en zonas rurales. Sólo en las regiones I y IX (ubicación ancestral de los pueblos Aymará y Mapuche respectivamente) se encuentran más del $20 \%$ de los hogares cuyo jefe de hogar pertenece a un pueblo indígena (Véase Cuadro 3) ${ }^{7}$.

Como se mostró en el Cuadro 1, la evidencia de las encuestas sugiere que las tasas de pobreza son mayores en la población indígena, situación que se atribuye generalmente a un menor capital humano entre sus miembros. De hecho, los años de educación de los adultos que trabajan es 7,3 años promedio en la población indígena, comparado con 9,5 años en la población no indígena (î.World" Bank, 2002): El nivel educacional del pueblo Mapuche es particularmente bajo, con $80 \%$ de los jefes de hogar con menos de 4 años de educación y menos de $3 \%$ de la población total con algún nivel de educación posterior a la enseñanza

5 La pregunta cambió desde: "Si usted es Chileno ¿Considera usted que pertenece a alguna de las siguientes culturas?” en 1992 a “¿Pertenece usted a alguno de los siguientes pueblos indígenas originarios?" en 2002.

6 Es posible que haya existido algo de subdeclaración también en el Censo de 1992 porque sólo se listaban 3 etnias como alternativa y porque la pregunta estaba dirigida a chilenos, por lo que algunos indígenas pueden no haberla respondido (Gundermann

7 En el momento del censo, Chile tenía 13 regiones, denominadas por número romanos desde el norte hacia el sur. La única excepción es la Región Metropolitana, que se ubica en el centro del país entre las regiones $\mathrm{V}$ y VI. 


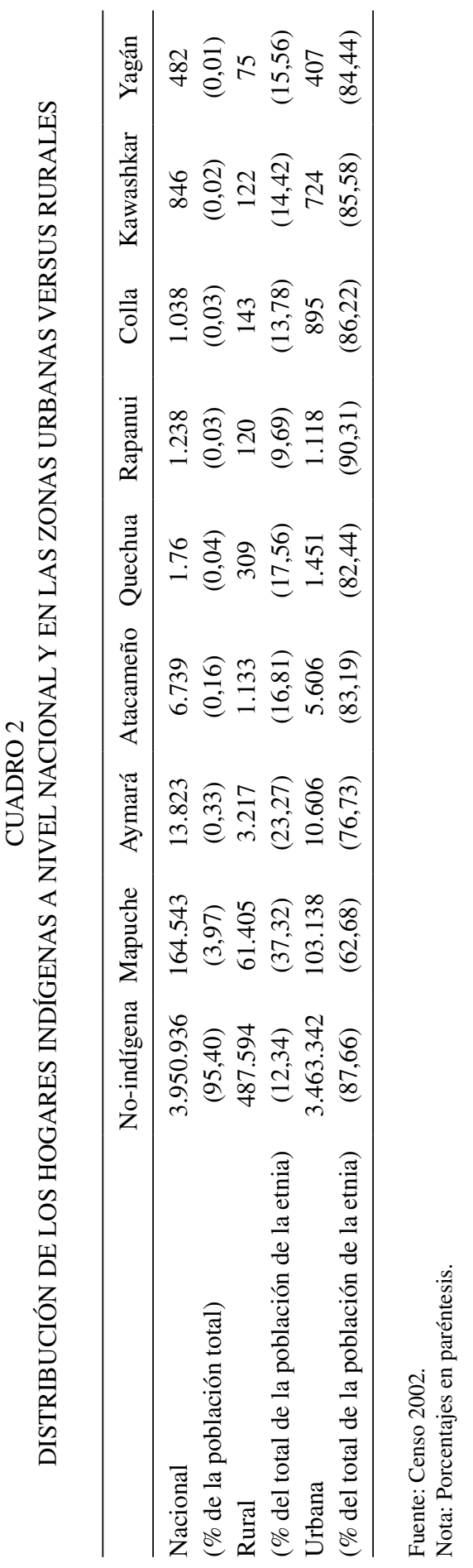




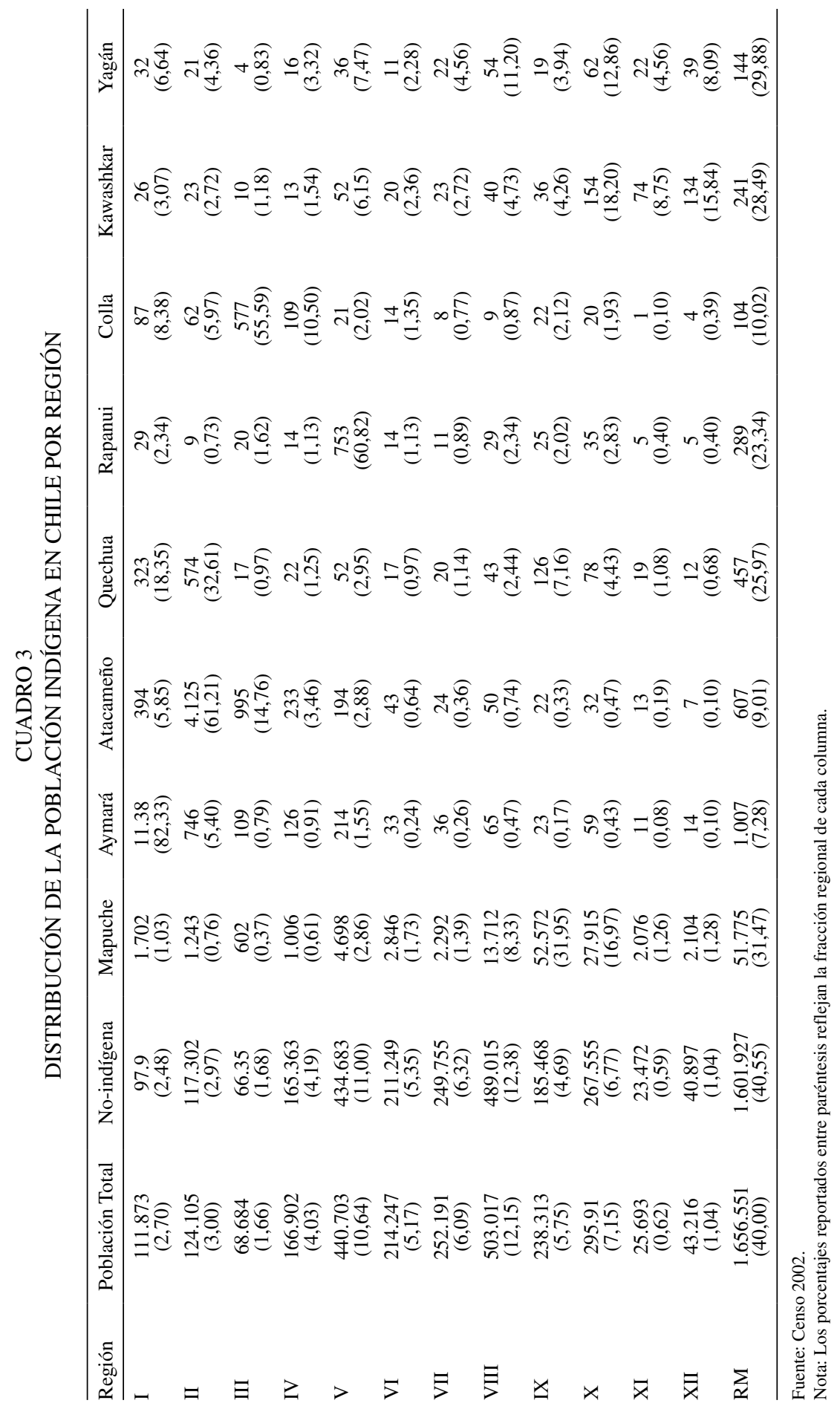


secundaria (WWorld Bank, 2002i). Adicionalmente, los niños de pueblos indígenas tienen rendimientos escolares bajos, con resultados entre 0,3 y 0,5 desviaciones estándar menores que los de niños no indígenas ( estado de salud la situación no es muy distinta y tanto la mortalidad y la diarrea infantil como la tuberculosis y la parasitosis son mucho mayores en la población

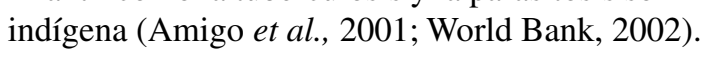

Finalmente, es importante mencionar que como parte de las políticas del gobierno chileno hacia los pueblos indígenas, se creó en 1992 la Corporación Nacional de Desarrollo Indígena (CONADI) con el objeto de compensar desigualdades y proteger los derechos de los pueblos indígenas. Hasta la fecha, gran parte del esfuerzo se ha concentrado en reformas y devolución de tierras junto al desarrollo de infraestructura productiva ${ }^{8}$. A pesar de estas iniciativas, los pueblos indígenas han manifestado su insatisfacción con los recursos canalizados a través de CONADI y persisten los reclamos respecto a la marginalización y la falta de oportunidades económicas. En respuesta, el gobierno anunció un "Pacto Social por la Multiculturalidad" que tiene por objetivo, para el período 2008-2010, mejorar la representación política de las comunidades indígenas, generar proyectos de desarrollo económico en zonas indígenas y crear mecanismos de consulta y participación de las comunidades indígenas en el caso de inversiones públicas y privadas que afecten sus comunidades (⿳⺈冂䒑

\section{Descripción DE los DATOS}

Para imputar ingreso utilizando la metodología descrita anteriormente se usan los datos de la encuesta CASEN 2003 ${ }^{9}$. Los datos de la encuesta incluyen etnicidad y características demográficas de los miembros del hogar, ingresos, características de la vivienda, propiedad de algunos bienes durables, acceso a servicios básicos y características de educación y salud. En el caso del ingreso, los datos son posteriormente ajustados por la Comisión Económica para América Latina y el Caribe (CEPAL) para corregir problemas de no respuesta y la sub o sobre representación de algunas categorías de ingreso en la muestra ${ }^{10}$.

La Casen 2003 encuestó a 68.153 hogares en 315 comunas de las 342 que existen en Chile, incluyendo 4.940 hogares con un jefe de hogar Mapuche, 1.012 con uno Aymará, 326 con uno Atacameño, 59 con uno Quechua, 9 con uno Rapanui, 19

8 El Fondo Tierra y Agua de la CONADI financia la compra de tierras, derechos de agua y proyectos de irrigación. El Fondo de Desarrollo entrega créditos, asistencia técnica y subsidios a las microempresas de indígenas.

9 Para mayores detalles metodológicos ver Pizzolito $(20-05)$

10 En el caso de no respuesta, se imputa el valor promedio del ingreso del grupo al cual pertenece el hogar de acuerdo a la intersección de varios criterios (región, educación, tipo de empleo, etc.). En el caso de la sub o sobre representación de ingreso, se realizan ajustes usando como referencia el sistema de cuentas nacionales del Banco Central de Chile. Si bien, los ajustes realizados por la CEPAL pueden generar algún grado de sesgo en los datos, Contreras (2003) argumenta que dicho sesgo sería mínimo. Para detalles adicionales ver 'ECLAC, IPEA E UNDP $(2002)$. 
por uno Colla, 13 con uno Kawashkar y solamente uno con un Yagán. La encuesta es representativa a nivel nacional, regional y para todas las áreas urbanas y todas las áreas rurales, pero no es representativa a nivel de etnias.

El Censo 2002, por otro lado, contiene datos de 4.112.838 hogares, de los cuales un $4.6 \%$ tienen un jefe de hogar perteneciente a un pueblo indígena ( $86 \%$ de ellos se identifica como Mapuche). Sin embargo, no contiene datos de ingreso. Como se mencionó previamente, es posible que exista algún error de medición en el número de personas en el Censo debido al cambio en la formulación de la pregunta. Sin embargo, es imposible determinar la dirección y la magnitud de ese potencial sesgo. Hay relativo consenso en que los datos de 2002 son más precisos que los de 1992, en los que había sobre-declaración de personas indígenas, y que las cifras son consistentes con otros análisis postcensales. En ese sentido, el usar el Censo de 2002 reduce e idealmente elimina el potencial sesgo respecto a usar el Censo de 1992.

Finalmente, para calcular las tasas de pobreza (indigencia) indígena se utiliza la línea de pobreza (indigencia) oficial del gobierno de Chile: 43.712 pesos chilenos (21.856 pesos chilenos) per cápita en zonas urbanas y 29.473 pesos chilenos (16.842 pesos chilenos) en zonas rurales (según datos de 'MīDEPLA $\overline{2} 0 \overline{0}$ i). Estas líneas de pobreza e indigencia se basan en los costos ponderados promedio de los requerimientos básicos de consumo alimenticio equivalente a alrededor de 2.176 calorías diarias.

\section{Resultados del Mapa de Pobreza Indígena}

Aplicando la metodología explicada anteriormente, se estimó en una primera etapa una regresión lineal para el ingreso per cápita del hogar. En cada una de las especificaciones de la regresión lineal se incluyeron variables demográficas, características de la vivienda y propiedad de activos junto a la interacción de algunas de estas variables. El propósito de estos modelos no es explicar causalmente los determinantes del ingreso de los hogares, sino que maximizar la fracción de la variación en los ingresos que puede ser explicada por variables observables.

Los resultados de la estimación de primera etapa para todos los hogares urbanos, todos los rurales, todos los hogares en el país y todos los de la Región Metropolitana de Santiago se presentan en el Cuadro B1 en el Apéndice B. Para resumir estos resultados, es importante mencionar que todos los coeficientes son estadísticamente significativos al $99 \%$ de confianza y todos los signos son los esperados. Por ejemplo, los bienes durables están asociados en forma positiva con mayor ingreso per cápita del hogar mientras que la falta de acceso a servicios básicos está asociada a menor ingreso per cápita en el hogar. El grado de ajuste de la regresión es relativamente alto para una regresión con datos seccionales y similar a los que se obtienen en estudios de mapas geográficos de pobreza

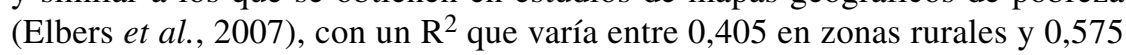
en la Región Metropolitana. 
El Cuadro 4 presenta las estimaciones de pobreza e indigencia para hogares urbanos y rurales por separado y nacional. Con el objeto de facilitar la interpretación de estos resultados, las Figuras 1, 2 y 3 muestran los estimadores para los 3 niveles de análisis. Se incluyen en las figuras el rango del intervalo de confianza al $90 \%$ de tal forma de poder comparar estadísticamente las diferencias entre los distintos pueblos indígenas bajo estudio y la población no indígena.

Tanto para hogares urbanos (Gráfico 1) como para hogares rurales (Gráfico 2) y para ambos juntos (Gráfico 3), la tasa de pobreza de los pueblos indígenas es mayor que la de la población no indígena al 90\% de confianza estadística. Para los hogares urbanos, por ejemplo, la tasa de pobreza estimada es de 34,3\% para los hogares indígenas y de $24,0 \%$ para los no indígenas. Incluso más, para cada pueblo indígena por separado, con la excepción del Yagán, la tasa de pobreza estimada es mayor que la de los no indígenas para los hogares urbanos y rurales combinados. Si bien los estimadores para el pueblo Yagán son más altos que para la población no indígena, el número de hogares Yaganes es tan pequeño que hace que el error estándar sea demasiado grande y, por lo tanto, la inferencia estadística sea más débil ${ }^{11}$.

Los estimadores son bastante precisos para los grupos indígenas más grandes en la población, con intervalos de confianza al 90\% menores a $\pm 1 \%$. Los dos pueblos indígenas más grandes, el Mapuche y el Aymará, tienen la mayor incidencia de pobreza: a un $90 \%$ de confianza estadística, sus tasas de pobreza son al menos siete puntos porcentuales más altas que la de la población no indígena. Los Mapuches y Aymarás también tienen niveles más altos de pobreza que el resto de los pueblos indígenas cuando se consideran los hogares urbanos y rurales en forma conjunta, con la excepción de los Quechua y los Colla para los que el intervalo de confianza se traslapa marginalmente.

Para hogares rurales, el pequeño tamaño de muestra dificulta hacer comparaciones similares. Por ejemplo, el tamaño muestral menor a 150 hogares para los pueblos Rapanui, Colla, Kawashkar y Yagán lleva a estimadores con errores estándar altos. Sin embargo, las estimaciones de pobreza rural sugieren un patrón de pobreza distinto entre los pueblos indígenas en Chile. Específicamente, los hogares rurales Atacameños, Rapanui, Kawashkar y Yagán experimentan pobreza en tasas similares a los hogares rurales no indígenas. Las diferencias en la pobreza de los Mapuches y los Aymará respecto a los no indígenas, por otro lado, se mantienen.

Observaciones similares se pueden hacer respecto a las estimaciones de indigencia. Para los hogares urbanos y para los urbanos y rurales tomados en conjunto, la población no indígena tiene tasas de indigencia menores que las de los pueblos indígenas, con la excepción de los Rapanui, Kawashkar y Yagán (Figuras 1 y 3). En las zonas rurales, cuando se consideran los pueblos indígenas por separado, sólo los Mapuches y los Aymarás presentan tasas más altas de indigencia al $90 \%$ de confianza estadística. Nuevamente, el pequeño tamaño muestral dificulta hacer inferencia estadística, si bien la diferencia entre

11 Así y todo, la tasa de pobreza es estimada con bastante precisión, con un intervalo de confianza menor $\mathrm{a} \pm 3,=5 \%$. 


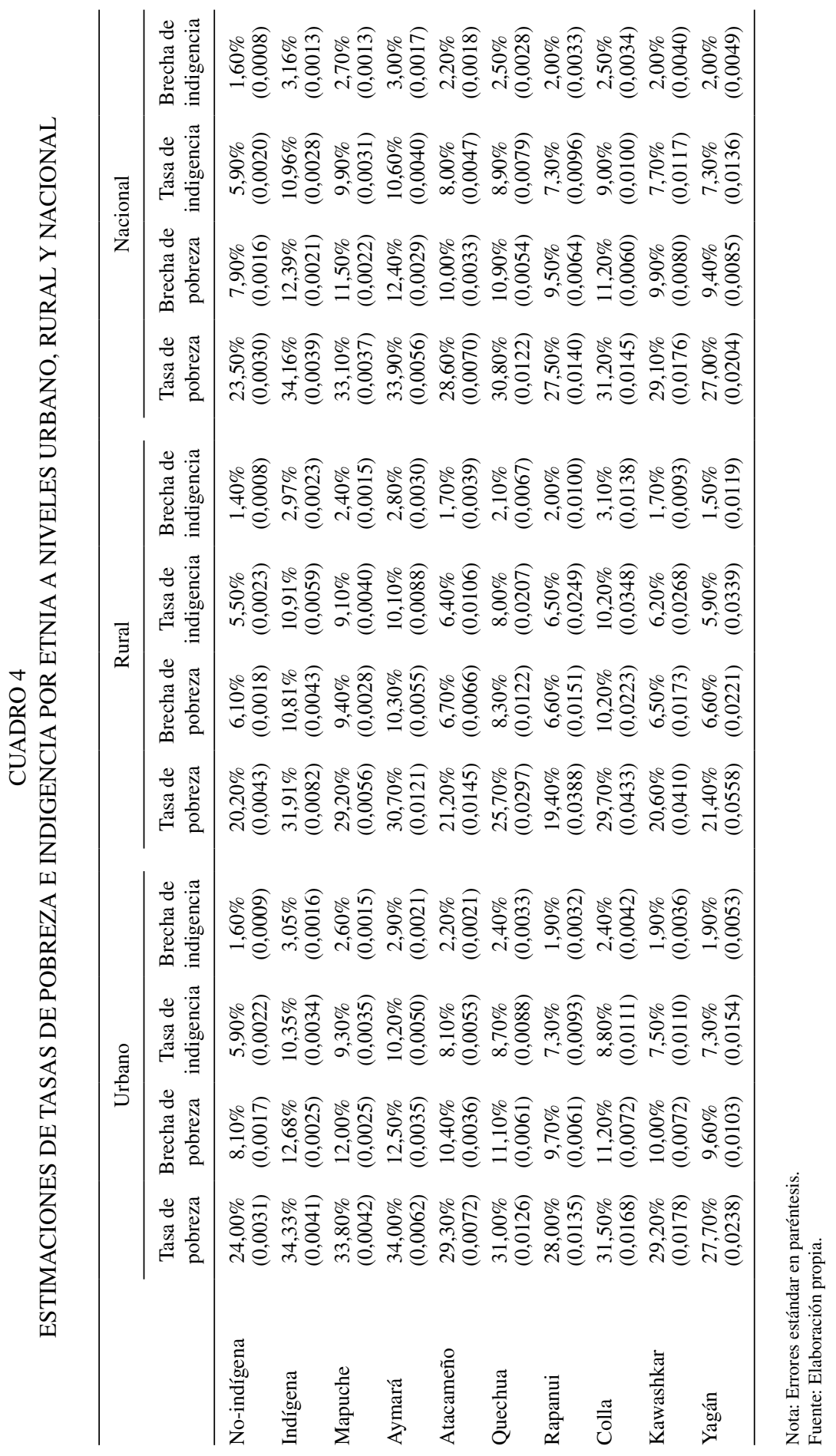


GRÁFICO 1

TASAS DE POBREZA E INDIGENCIA EN CHILE URBANO

CON INTERVALOS DE CONFIANZA AL 90\%

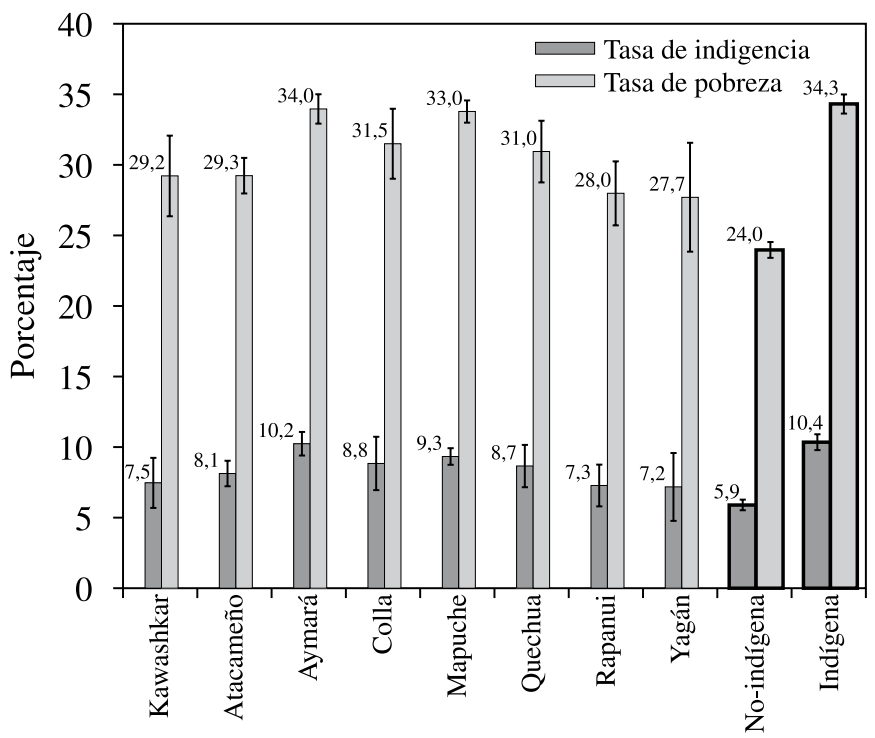

Fuente: Elaboración propia.

GRÁFICO 2

TASAS DE POBREZA E INDIGENCIA EN CHILE RURAL

CON INTERVALOS DE CONFIANZA AL 90\%

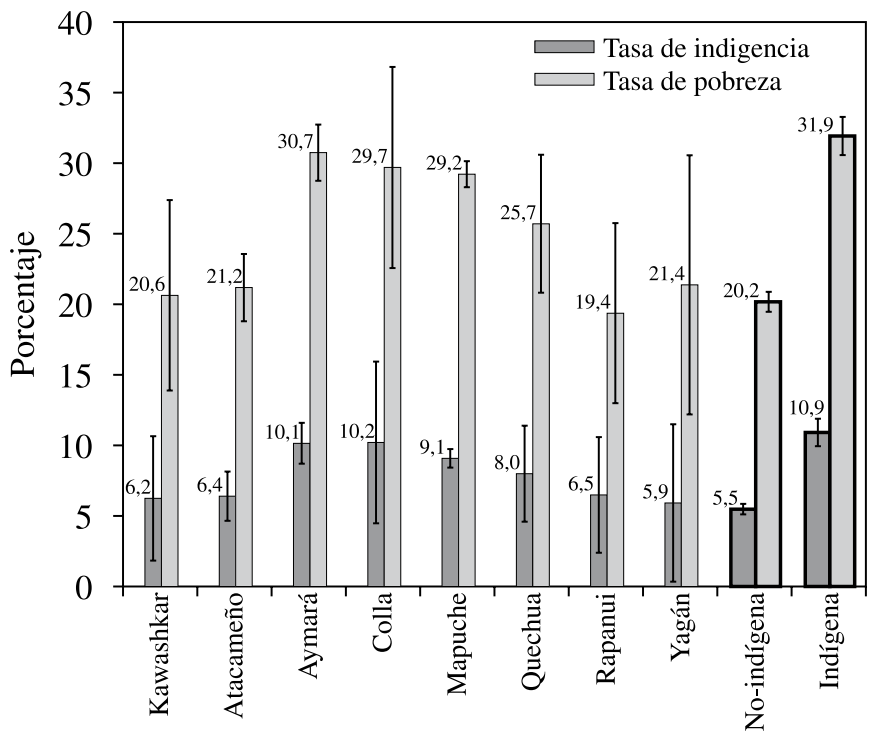

Fuente: Elaboración propia. 


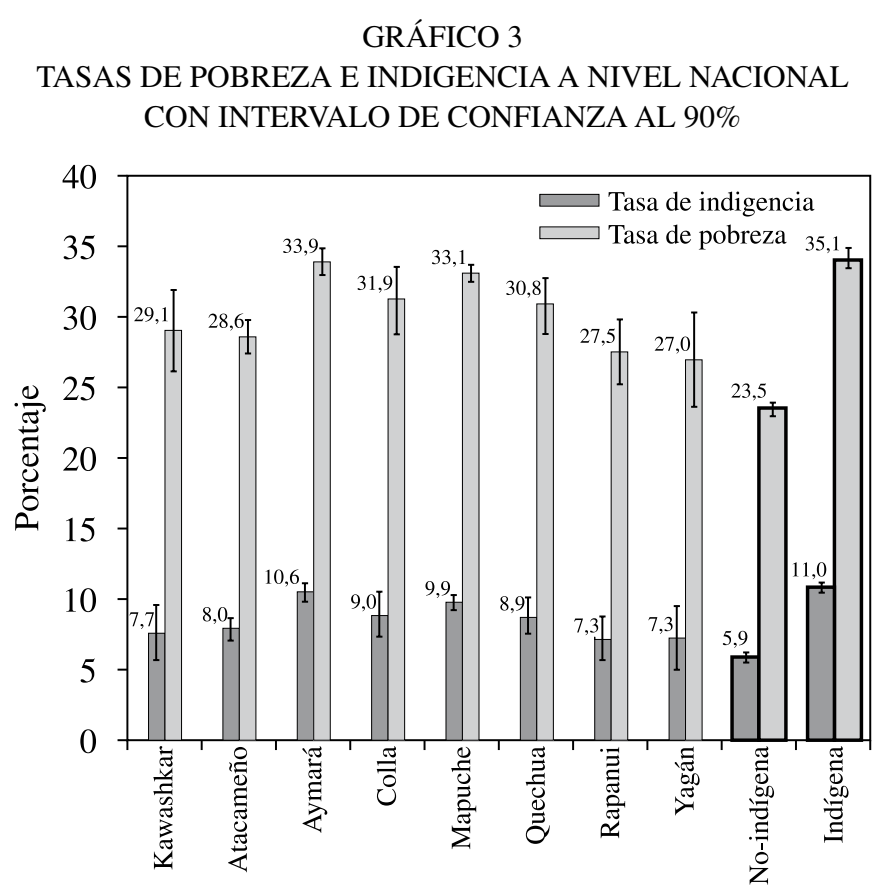

Fuente: Elaboración propia.

la tasa de indigencia de la población no indígena versus la indígena al considerar todos los pueblos en forma conjunta es similar a la diferencia existente en zonas urbanas, lo cual sugiere que las tasas de indigencia son en realidad mayores en los pueblos indígenas.

La profundidad de la pobreza también puede evaluarse a través de la medida de brecha de pobreza ${ }^{12}$. Tal como se muestra en el Cuadro 4 , transferir alrededor del $8 \%$ del total de ingreso disponible de los chilenos no indígenas a los que viven bajo la línea de pobreza es suficiente para erradicar completamente la pobreza, mientras que 12,4\% del ingreso disponible de los Aymará debe ser redistribuido para eliminar la pobreza en dicho pueblo indígena. Con un nivel de $90 \%$ de confianza estadística, la brecha de pobreza para los chilenos no indígenas es menor que la de cualquier otro grupo étnico con la excepción de los Yagán. Las mayores brechas de pobreza corresponden a los pueblos Colla y Aymará.

Las brechas de pobreza están fuertemente correlacionadas con las brechas de indigencia para cada pueblo indígena. A nivel nacional, la brecha de indigencia de la población no indígena es menor que la de cada uno de los pueblos indígenas existentes. De esta forma, los resultados muestran que los pueblos indígenas experimentan mayor incidencia y mayor profundidad de la pobreza que la población no indígena en Chile.

12 La brecha de pobreza es medida utilizando el estadístico de Foster-Greer-Thorbecke. 


\section{CUADRO 5 \\ MEDIDAS DE POBREZA E INDIGENCIA POR REGIÓN \\ (Porcentaje)}

\begin{tabular}{llcc}
\hline Región & Nombre de la región & Tasa de pobreza & Tasa de indigencia \\
\hline I & Tarapacá & 18,46 & 3,21 \\
II & Antofagasta & 11,22 & 3,24 \\
III & Atacama & 24,85 & 8,09 \\
IV & Coquimbo & 21,54 & 5,29 \\
V & Valparaíso & 19,40 & 4,65 \\
VI & O’Higgins & 19,22 & 3,97 \\
VII & Maule & 23,10 & 5,61 \\
VII & Biobío & 28,01 & 8,44 \\
IX & Araucanía & 29,71 & 9,47 \\
X & Los Lagos & 21,83 & 4,80 \\
XI & Aysén & 14,19 & 4,22 \\
XII & Magallanes & 12,29 & 2,45 \\
XIII & Región Metropolitana & 13,08 & 2,85 \\
\hline
\end{tabular}

Fuente: MIDEPLAN - CASEN 2003.

Obviamente, mayores tasas de pobreza de algunos pueblos indígenas pueden reflejar tanto etnicidad como geografía, porque la pobreza es distinta en cada región del país y los pueblos indígenas no están distribuidos en forma geográficamente homogénea sino que se concentran en algunas regiones. Por ejemplo, tal como se muestra en el Cuadro 5, la pobreza en la región IX (donde se encuentra la mayor parte de la tierra ancestral del pueblo Mapuche) es 29,7\% mientras que en la región II (la tierra ancestral del pueblo Atacameño) es de 11,2\%. Es posible entonces que mayores tasas de pobreza simplemente reflejen distintas oportunidades económicas entre regiones más que distintas oportunidades económicas para los pueblos indígenas. Para considerar esta posibilidad se utilizan dos estrategias ${ }^{13}$. Primero, se examina la pobreza y la indigencia por pueblo indígena en la Región Metropolitana de Santiago que es la región en la cual todos los pueblos indígenas están bien representados, por lo que al restringir la muestra a Santiago es posible aislar en mejor medida las diferencias étnicas en pobreza e indigencia ${ }^{14}$. Existen diferencias importantes entre la población indígena y algunos pueblos indígenas en esta zona geográfica más restringida (Cuadro 6 y Gráfico 4). Por ejemplo, la tasa de pobreza para los Mapuches es casi 10 puntos porcentuales mayor que la de la población no indígena, muy similar a la situación existente en el país como

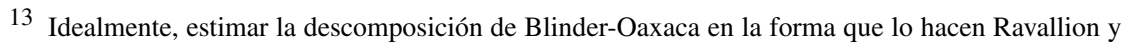
Wodon 1 1999debido a la característica del proceso generador de los datos de pobreza para que sean representativos y el objetivo de las regresiones de primera etapa en los mapas de pobreza, no es posible hacerlo.

14 Por ejemplo, más Aymarás viven en Santiago que en la Región I, donde este pueblo tienen sus raíces históricas. En forma similar, hay tantos Mapuches viviendo en Santiago como en la Región IX. 
CUADRO 6

MEDIDAS DE POBREZA E INDIGENCIA POR ETNIA

EN SANTIAGO DE CHILE

\begin{tabular}{lcccc}
\hline & $\begin{array}{c}\text { Tasa } \\
\text { de pobreza }\end{array}$ & $\begin{array}{c}\text { Brecha } \\
\text { de pobreza }\end{array}$ & $\begin{array}{c}\text { Tasa } \\
\text { de indigencia }\end{array}$ & $\begin{array}{c}\text { Brecha } \\
\text { de indigencia }\end{array}$ \\
\hline No-indígena & $18,60 \%$ & $5,90 \%$ & $4,00 \%$ & $1,00 \%$ \\
& $(-0,0058)$ & $(-0,0026)$ & $(-0,0027)$ & $(-0,0009)$ \\
Indígena & $28,80 \%$ & $9,98 \%$ & $7,57 \%$ & $2,16 \%$ \\
& $(-0,0072)$ & $(-0,0043)$ & $(-0,0055)$ & $(-0,0024)$ \\
Mapuche & $28,10 \%$ & $9,30 \%$ & $6,50 \%$ & $1,66 \%$ \\
& $(-0,0083)$ & $(-0,0041)$ & $(-0,0046)$ & $(-0,0015)$ \\
Aymará & $16,20 \%$ & $5,20 \%$ & $3,50 \%$ & $0,92 \%$ \\
& $(-0,0129)$ & $(-0,0053$ & $(-0,0071)$ & $(-0,0024)$ \\
Atacameño & $16,80 \%$ & $5,20 \%$ & $3,40 \%$ & $0,87 \%$ \\
& $(-0,0159)$ & $(-0,0063)$ & $(-0,0086)$ & $(-0,0029)$ \\
Quechua & $22,30 \%$ & $7,20 \%$ & $5,00 \%$ & $1,29 \%$ \\
& $(-0,0189)$ & $(-0,0084)$ & $(-0,0119)$ & $(-0,0038)$ \\
Rapanui & $22,20 \%$ & $7,50 \%$ & $5,60 \%$ & $1,44 \%$ \\
& $(-0,0257)$ & $(-0,0104)$ & $(-0,0155)$ & $(-0,0054)$ \\
Colla & $14,70 \%$ & $4,30 \%$ & $2,60 \%$ & $0,66 \%$ \\
& $(-0,035)$ & $(-0,0129)$ & $(-0,0169)$ & $(-0,0056)$ \\
Kawashkar & $22,70 \%$ & $7,00 \%$ & $4,40 \%$ & $1,03 \%$ \\
& $(-0,0298)$ & $(-0,0121)$ & $(-0,016)$ & $(-0,0049)$ \\
Yagán & $17,40 \%$ & $5,70 \%$ & $4,10 \%$ & $1,09 \%$ \\
& $(-0,0329)$ & $(-0,013)$ & $(-0,0196)$ & $(-0,0064)$ \\
\hline
\end{tabular}

Fuente: Elaboración propia.

Nota: Errores estándar en paréntesis.

\section{GRÁFICO 4}

TASAS DE POBREZA E INDIGENCIA EN LA REGIÓN XIII (SANTIAGO) CON INTERVALO DE CONFIANZA AL 90\%

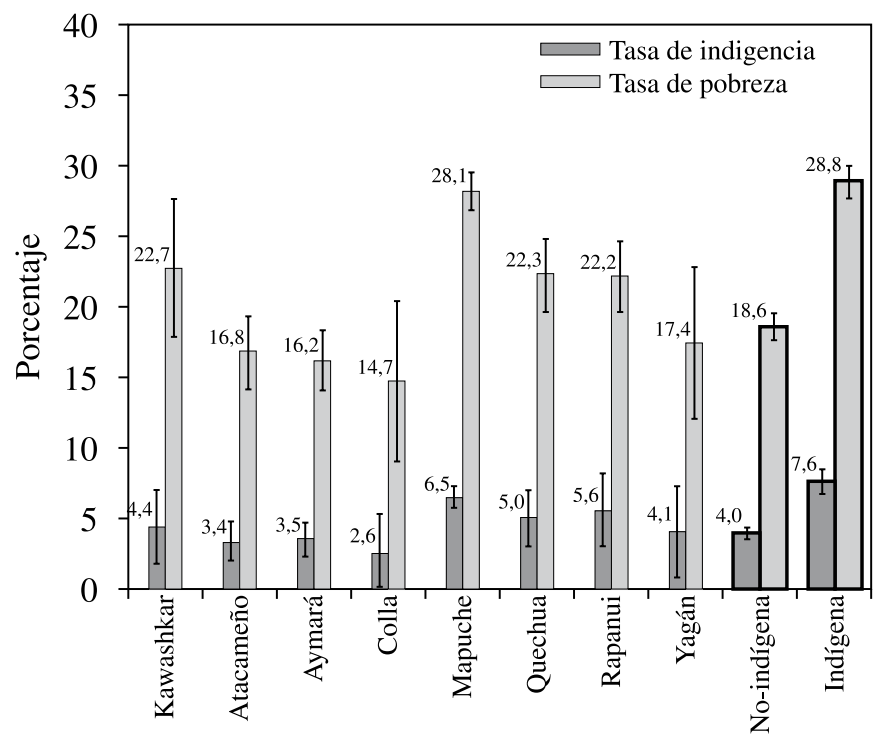

Fuente: Elaboración propia. 


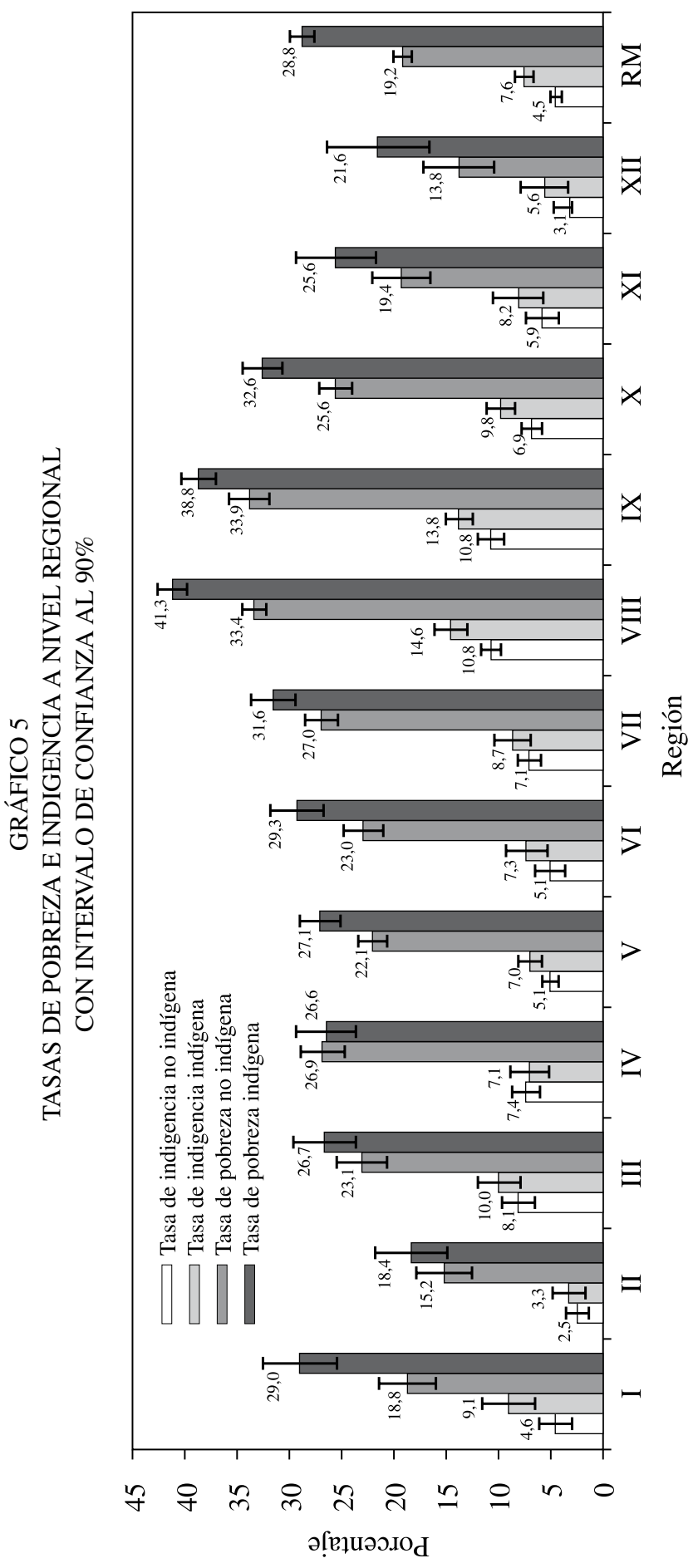

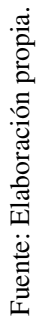




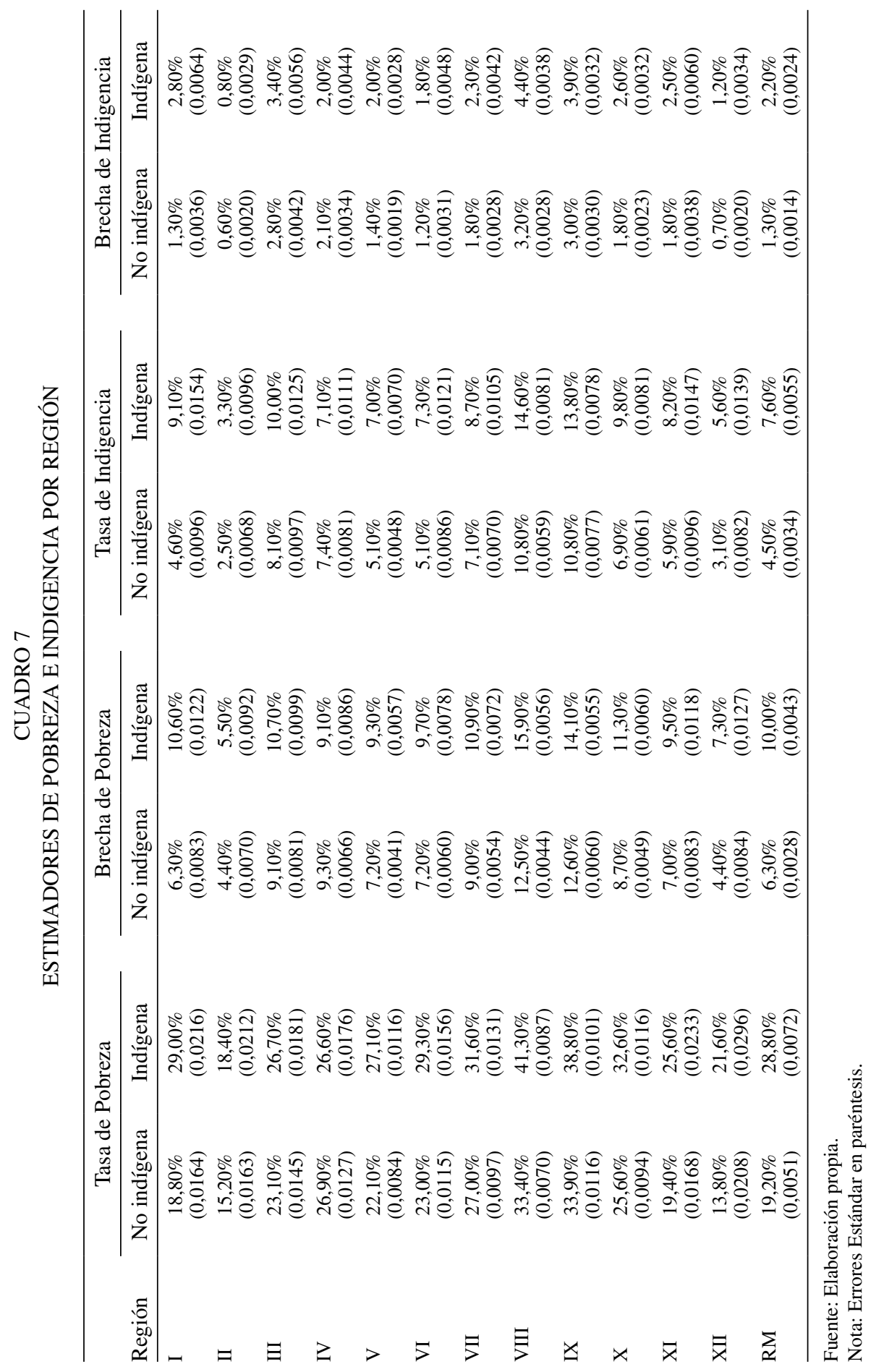


un todo. La tasa de pobreza del pueblo Quechua es también significativamente alta. Sin embargo, un patrón distinto surge para los otros pueblos indígenas: los estimadores de la tasa de pobreza para los Aymará, los Atacameños, los Colla y los Yagán son menores que la tasa de pobreza de los no indígenas, si bien esta diferencia no es estadísticamente significativa al 90\% de confianza.

Segundo, se comparan la pobreza y la indigencia para cada población indígena como un todo respecto a la población no indígena, en cada una de las 13 regiones del país. El Cuadro 7 y el Gráfico 5 muestran una discrepancia estadística significativa entre las poblaciones indígena y no indígena en ocho de las 13 regiones de Chile. En la región IX, por ejemplo, la tasa de pobreza estimada para la población indígena es de $38,8 \%$, cinco puntos porcentuales mayor que la da la población no indígena. En la Región I, los estimadores correspondientes son $29 \%$ para los indígenas y 18,8\% para los no indígenas. El pequeño tamaño muestral en la Regiones XI y XII producen errores estándar relativamente grandes, por lo que en estos dos casos los intervalos de confianza al $90 \%$ se traslapan. Las tasas de pobreza no son estadísticamente diferentes en las regiones II, III y IV. En la Región II, las tasas de pobreza relativamente bajas para ambos grupos es probablemente el resultado del alto crecimiento económico asociado a la minería del cobre. En la Regiones III y IV, las tasas de pobreza relativamente altas son compartidas tanto por los indígenas como por los no indígenas.

Las diferencias entre la población indígena y la no indígena en términos de indigencia son aún más pronunciadas en la mayoría de las regiones que las diferencias en pobreza. Por ejemplo, la tasa de indigencia estimada para la población indígena en la Región I $(9,1 \%)$ es casi el doble que la estimada para la población no indígena (4,6\%). Grandes diferencias en las tasas de indigencia se observan también en las Regiones VIII, X, XI, XII y RM. La única región en la cual la población no indígena muestra una estimación más alta de indigencia que la de la población indígena es la Región IV, pero esta diferencia está lejos de ser estadísticamente significativa.

Las brechas estimadas de pobreza e indigencia al nivel de cada región muestran que, con la excepción de las Regiones III y IV, la población indígena no sólo es afectada por mayores tasas de pobreza sino que también por una profundidad de pobreza más aguda. Una vez más, las Regiones I, VIII, XII y RM aparecen como las áreas en el país que contienen las mayores diferencias entre los dos tipos de población.

\section{Conclusiones}

El gobierno chileno ha realizado algunos esfuerzos importantes en la dirección de identificar mejor a los hogares pobres para la implementación de sus políticas sociales, es así como la ficha CAS-2 se eliminó y fue reemplazada por la Ficha de Protección Social (FPS). Mientras que la ficha CAS-2 ponía un fuerte énfasis en las características de la vivienda y en la propiedad de activos para identificar pobreza, la nueva ficha FPS evalúa a los hogares de acuerdo a su 
potencial de generar ingresos. Para ello considera la estabilidad de los ingresos, los niveles educacionales de los miembros del hogar, su experiencia laboral, su edad, su estado de salud, la existencia de discapacidades, el número de personas en el hogar, la ubicación geográfica y el nivel de desempleo en dicha zona. Estos nuevos criterios probablemente van a mejorar la focalización de los subsidios que entrega el Estado, si bien no consideran la pertenencia a un pueblo indígena dentro de las variables relevantes.

En este trabajo, se muestra que existe una notoria disparidad en las tasas de pobreza e indigencia de los pueblos indígenas respecto a las de la población no indígena en todos los niveles espaciales considerados: nacional, urbano y rural. Por ejemplo, la tasa de pobreza de hogares indígenas a nivel nacional es de alrededor de 10 puntos porcentuales mayor que la de hogares no indígenas. De hecho, con la excepción del pueblo Yagán (para el cual la muestra es demasiado pequeña), la tasa de pobreza es estadísticamente más alta con un $90 \%$ de confianza para cada uno de los pueblos indígenas respecto a la población no indígena del país. Las tasas de pobreza son particularmente altas para los pueblos Mapuche y Aymará, con alrededor de un tercio de los hogares viviendo bajo la línea de la pobreza.

Adicionalmente, altas tasas de pobreza están fuertemente correlacionadas con mayor profundidad de la pobreza. Por ejemplo, la brecha de pobreza estimada es $7,9 \%$ a nivel nacional para los chilenos no indígenas, comparada con $12,4 \%$ para los Aymará, 11,5\% para los Mapuche y 11,2\% para los Colla. Las tasas de indigencia siguen patrones similares y las estimaciones para la brecha de indigencia de los Aymará es casi el doble que la de los no indígenas.

Este patrón de resultados es bastante general y se mantiene al considerar regiones geográficas específicas. Por ejemplo, las tasas de pobreza de los pueblos indígenas son estadísticamente más altas en ocho de las trece regiones de Chile (y muy cercana a serlo en otras dos). Estos resultados sugieren que la variación étnica en la distribución geográfica de la población no es suficiente para explicar la incidencia de la pobreza en los pueblos indígenas. Por el contrario, la evidencia empírica muestra que la pertenencia a un pueblo indígena es un fuerte predictor de pobreza e indigencia, lo cual puede reflejar que los miembros de pueblos indígenas experimentan un acceso diferencial a oportunidades económicas a pesar de las políticas implementadas por CONADI. Por ello, incluir etnicidad dentro de los criterios para identificar pobreza puede mejorar en forma significativa la focalización y los resultados de la política social del gobierno. En ese contexto, es relevante poder mejorar la precisión con la cual se cuantifica la población indígena en Chile. El cambio en la forma de preguntar entre el Censo de 1992 y el de 2002, junto a la realización de un estudio y testeo previo en el caso del Censo de 2002, representan un avance importante. Sin embargo, es posible mejorar la precisión a partir de la utilización de múltiples preguntas tanto en el Censo como en la Casen que permitan identificar mejor la población indígena en Chile y su distribución territorial. 


\section{REFERENCIAS}

Agostini, C. A. y P. H. Brown (2010a), "Local Distributional Effects of Government Cash Transfers in Chile", Review of Income and Wealth, (in press).

Agostini, C. A. y P. H. Brown (2010b), "Inequality at Low Levels of Aggregation in Chile", Review of Development Economics, 14(2): 213-226.

Agostini, C. A., P. H. Brown y A. Roman (2010), "Poverty and Inequality Among Ethnic Groups in Chile", World Development, (in press).

Amigo, H., P. Bustos, C. Leone y M. E. Radrigán (2001), "Growth Deficits in Chilean School Children", Journal of Nutrition, 131: 251-254.

Beyer, H. (1997), "Distribución del Ingreso: Antecedentes para la Discusión”, Estudios Públicos, 65: 5-58.

Bigman, D. y H. Fofack (2000), Geographical Targeting for Poverty Alleviation. Methodology and Applications. Washington, DC. World Bank.

Contreras, D. (2003), "Poverty and Inequality in a Rapid Growth Economy: Chile 199096", Journal of Development Studies, 39(3): 181-200.

Contreras, D., O. Larrañaga, J. Litchfield y A.Valdés (2001), "Poverty and Income Distribution in Chile 1987-1998: New Evidence", Cuadernos de Economía (Latin American Journal of Economics), 114: 191-208.

Datt, G. y M. Ravallion (1993), Regional Disparities, Targeting, and Poverty in India. Washington, DC. World Bank.

Demombynes, G. y B. Özler (2005), "Crime and Local Inequality in South Africa", Journal of Development Economics, 76(2): 265-292.

Economic Commission for Latin America and the Caribbean, Instituto de Pesquisa Econômica Aplicada, and United Nations Development Program (2002), Meeting the Millennium Poverty Reduction Target in Latin America and the Caribbean, Santiago: ECLAC.

Elbers, C., J. O. Lanjouw y P. Lanjouw (2003), "Micro-Level Estimation of Poverty and Inequality", Econometrica, 71(1): 355-364.

Elbers, C., T. Fujii, P. Lanjouw, B. Özler y W. Yip (2007), "Poverty Alleviation Through Geographic Targeting: How Much Does Disaggregation Help?", Journal of Development Economics, 83: 198-213.

Gobierno de Chile (2004), "Política de Nuevo Trato con Los Pueblos Indígenas. Derechos Indígenas, Desarrollo con Identidad y Diversidad Cultural.” Mimeo. URL: http:///www.mapuche.info/mapuint/newtreat $040400 . p \mathrm{df}$.

Gundermann, H., J. I. Vergara y G. R. Foerster (2005), “Contar a los Indígenas en Chile: Autoadscripción Étnica en la Experiencia Censal de 1992 y 2002", Estudios Atacameños, 30.

Hentschel, J., J. O. Lanjouw, P. Lanjouw y J. Poggi (1999), “Combining Census and Survey Data to Trace the Spatial Dimensions of Poverty: A Case Study from Ecuador", World Bank Economic Review, 14(1); 147-165.

Larrañaga, O. (2001), "Distribución de Ingresos en Chile: 1958-2001", Documento de Trabajo $\mathrm{N}^{\circ}$. 178, Departamento de Economía, Universidad de Chile.

Malinowski, M.(2008), “Chile's Reformed Indigenous Policies Receive Mixed Reviews." Santiago Times, 04/03/2008.

McEwan, P. (2004), "The Indigenous Test Score Gap in Bolivia and Chile", Economic Development and Cultural Change, 53: 157-190.

MIDEPLAN (2005), "Marco Metodólogico-CASEN 2003”, Departamento Información Social, División Social, MIDEPLAN, Gobierno de Chile. 
Pizzolito, G. (2005), "Poverty and Inequality in Chile: Methodological Issues and a Literature Review", CEDLAS, Working Paper No. 20.

Ravallion, M. y Q. Wodon (1999a), Evaluating a Targeted Social Program When Placement Is Decentralized, Washington D.C.: The World Bank.

Ravallion, M. y Q. Wodon (1999b), "Poor Areas or Only Pooer People", Journal of Regional Science, 39(4).

Sanderson, J. (2005), "La Poblacion Indígena en Chile: El Censo y las Encuestas Territoriales" en CEPAL (eds), "Pueblos Indígenas y Afrodescendientes de America Latina y el Caribe: Informacion Sociodemográfica para Políticas y Programas", Santiago, pp. 109-124.

Valdés, A. (1999), "Pobreza y Distribución del Ingreso en una Economía de Alto Crecimiento: Chile, 1987-1995”, Estudios Públicos, 75: 5-47.

Valenzuela, R. (2003), “Inequidad, Ciudadanía y Pueblos Indígenas en Chile”, CEPAL, Serie Políticas Sociales 76.

World Bank (2002), "Indigenous Peoples in Chile. Current Situation and Policy Issues" in Chile: Poverty and Income Distribution in a High Growth Economy. Mimeo. 


\section{ApÉNDICE A}

\section{Metodología}

En la primera etapa, se estima un modelo para el ingreso per cápita del hogar $h\left(Y_{h}\right)$ asociado a un cluster étnico $c$ y a un conjunto de características observables $\left(X_{h}\right)$ :

(A1) $\ln Y_{h c}=E\left[\ln Y_{h c} / X_{h c}\right]+u_{h c}=X_{h c} \beta+u$

El vector de errores $u$ se asume que tiene una distribución con media cero y matriz varianza-covarianza $\Sigma: f(0, \Sigma)$. Para permitir algún grado de correlación dentro de cada cluster se utiliza la siguiente especificación:

$$
u_{h c}=\eta_{c}+\varepsilon_{h c}
$$

donde $\eta$, el componente de cluster, y el error idiosincrático $\varepsilon$ son independientes entre sí y no están correlacionadas con las variables observables $X_{h c}$.

Este modelo se estima con los datos de la encuesta de hogares y como resultado de la estimación se obtiene un conjunto de parámetros estimados del modelo: el vector $\beta$, la matriz varianza-covarianza asociada a este vector y los parámetros que describen la distribución de los errores.

En la segunda etapa, se combinan los parámetros estimados en la primera etapa con las características observables de cada individuo y hogar en el censo para generar valores predichos del logaritmo del ingreso y los errores relevantes. Para estos efectos se utiliza un método de bootstrap que simula los valores del ingreso de cada hogar o de cada individuo. Los valores simulados se basan en la predicción de los ingresos y los términos de error $\eta \mathrm{y} \varepsilon$ :

$$
\text { (A3) } \hat{Y}_{h c}=\exp \left(X_{h c} \hat{\beta}+\hat{\eta}_{c}+\hat{\varepsilon}_{h c}\right)
$$

donde, para cada hogar, los dos componentes del término de error son obtenidos de la distribución empírica descrita por los parámetros en la primera etapa ${ }^{15}$. De igual forma, los coeficientes $\hat{\beta}$ se obtienen de la distribución descrita por los estimadores de $\beta$ en la primera etapa y la matriz varianza-covarianza asociada a ellos ${ }^{16}$.

Por último, para generar los indicadores de pobreza, se utiliza el set completo de valores simulados de $\hat{Y}_{h c}$ para calcular el valor esperado de dichos indicadores por pueblo indígena. El procedimiento se repite $n$ veces, obteniendo un nuevo set de coeficientes $\beta$ y errores para cada simulación. Para cada pueblo indígena, se calcula la media y la desviación estándar del indicador de pobreza sobre el total de

\footnotetext{
15 Para los dos componentes del error, hogar y cluster, se utiliza una distribución normal.

16 Utilizando el Teorema del Límite Central, se asume que la distribución del vector $\beta$ converge a una distribución normal multivariada.
} 
simulaciones, que constituyen las estimaciones punto del indicador y los errores estándar de estos estimadores respectivamente.

Es así como en este trabajo se utilizan simulaciones de Montecarlo para calcular el valor esperado de la tasa de pobreza de cada etnia $(P)$, condicional en la regresión de la primera etapa, la varianza generada por el componente indiosincrático del ingreso per cápita de los hogares y el vector de gradientes. Cada simulación obtiene 200 vectores de términos de error de la distribución estimada en la primera etapa, con cada uno de estos vectores se calcula un valor para $P\left(\hat{P}_{d}^{E}\right.$ en la ecuación siguiente $)$ y luego el valor esperado simulado para $P$ ( $\tilde{P}^{E}$ en la ecuación siguiente) es la media para las 250 réplicas:

(A4) $\tilde{P}^{E}=\frac{1}{250} \sum_{d=1}^{250} \hat{P}_{d}^{E}$

De la misma manera, la varianza de $P$ es estimada usando:

(A5) $\quad \hat{V}_{\text {modelo }}=\frac{1}{250} \sum_{d=1}^{250}\left(\hat{P}_{d}^{E}-\tilde{P}^{E}\right)^{2}$. 


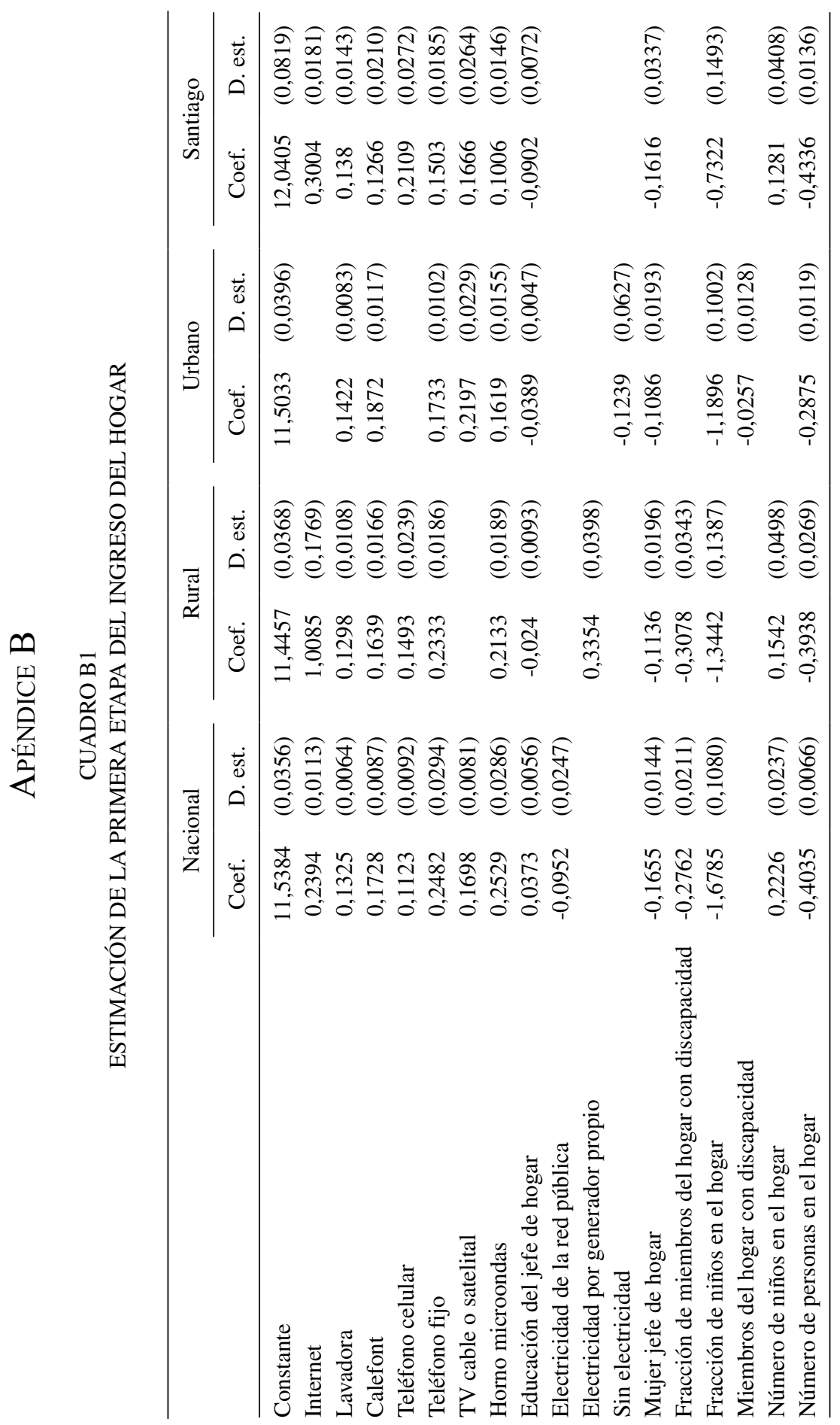




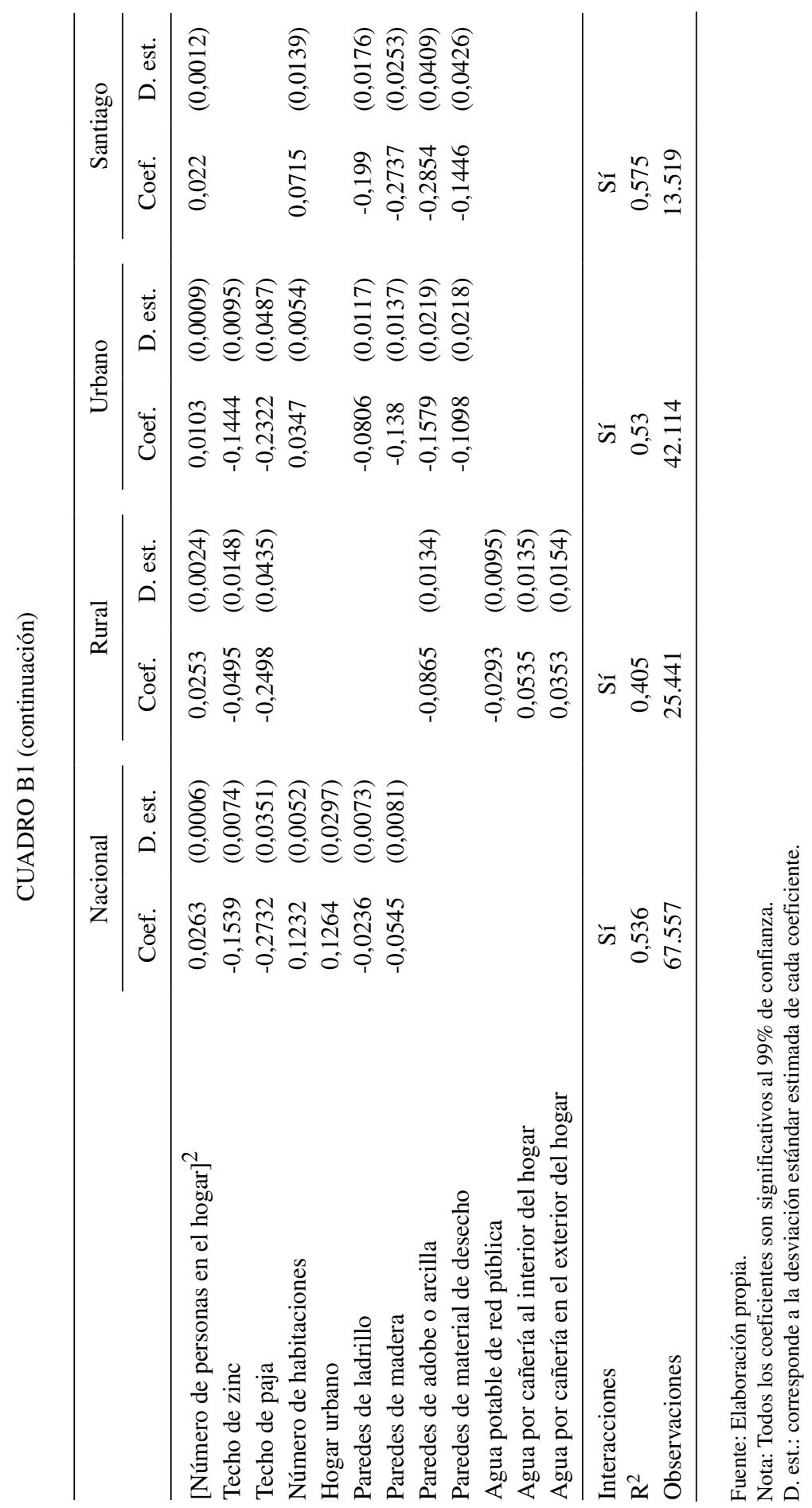

